\title{
Comparison of telemedicine with in-person care for follow-up after elective neurosurgery: results of a cost-effectiveness analysis of 1200 patients using patient-perceived utility scores
}

\author{
Sumit Thakar, MCh, ${ }^{1}$ Niranjana Rajagopal, DNB, ${ }^{1}$ Subramaniyan Mani, MTech, ${ }^{2}$ \\ Maya Shyam, PGDM, ${ }^{3}$ Saritha Aryan, MS, MCh, ${ }^{1}$ Arun S. Rao, DNB, ${ }^{1}$ Rakshith Srinivasa, MCh, ${ }^{1}$ \\ Dilip Mohan, MS, MCh, DNB, ${ }^{1}$ and Alangar S. Hegde, MCh, PhD ${ }^{1}$
}

${ }^{1}$ Department of Neurological Sciences, ${ }^{2} \mathrm{Hospital}$ Management Information System, and ${ }^{3}$ Finance and Accounts, Sri Sathya Sai Institute of Higher Medical Sciences, Bangalore, India

OBJECTIVE The utility of telemedicine (TM) in neurosurgery is underexplored, with most of the studies relating to teletrauma or telestroke programs. In this study, the authors evaluate the cost-effectiveness of TM consultations for followup care of a large population of patients who underwent neurosurgical procedures.

METHODS A decision-analytical model was used to assess the cost-effectiveness of TM for elective post-neurosurgical care patients from a predominantly nonurban cohort in West Bengal, India. The model compared TM care via a nodal center in West Bengal to routine, in-person, per-episode care at the provider site in Bangalore, India. Cost and effectiveness data relating to 1200 patients were collected for a 52-month period. The effectiveness of TM care was calculated using efficiency in terms of the percentage of successful TM consultations, as well as patient-perceived utility values for overall experience of the type of health care access that they received. Incremental cost-effectiveness ratio (ICER) analysis was done using the 4-quadrant charting of the cost-effectiveness plane. One-way sensitivity and tornado analyses were performed to identify thresholds where the care strategy would change.

RESULTS The overall utility for the 3 TM scenarios was found to be higher (89\%) than for the utility of routine care (80\%). TM was found to be more cost-effective (Indian rupee [INR] 2630 per patient) compared to routine care (INR 6848 per patient). The TM strategy "dominates" that of routine care by being more effective and less expensive (ICER value of $-39,400$ INR/unit of effectiveness). Sensitivity analysis revealed that cost-effectiveness of TM was most sensitive to changes in the number of TM patients, utility and success rate of TM, and travel distance to the TM center.

CONCLUSIONS TM care dominates the in-person care strategy by providing more effective and less expensive followup care for a remote post-neurosurgical care population in India. In the authors' setting, this benefit of TM is sustainable even if half the TM consultations turn out to be unsuccessful. The viability of TM as a cost-effective care protocol is attributed to a combination of factors, like an adequate patient volume utilizing TM, patient utility, success rate of TM, and the patient travel distance.

https://thejns.org/doi/abs/10.3171/2018.2.FOCUS17543

KEYWORDS cost-effectiveness; telemedicine; postoperative; follow-up; neurosurgery

$\mathrm{T}$ HE application of telemedicine (TM) in neurosurgical care has been underexplored, with most of the existing studies being related to telemedicine for trauma (teletrauma) $)^{3,4,17}$ and stroke (telestroke). ${ }^{24,29}$ In a lower-middle-income economy like India, where a majority of the population resides in rural areas and has limited access to specialty services such as neurosurgery, ${ }^{25}$ the need for evaluating the cost-effectiveness of TM becomes all the more important. While there have been many feasibility studies in TM across specialties, ${ }^{2}$ there are only a few studies that have addressed the economic utility of TM. In one of the largest TM-related studies in neurosurgery, we

ABBREVIATIONS CEA = cost-effectiveness analysis; CGHS = Central Government Health Scheme; ICER = incremental cost-effectiveness ratio; INR = Indian rupee; TM = telemedicine.

ACCOMPANYING EDITORIAL DOI: 10.3171/2018.2.FOCUS1889.

SUBMITTED August 22, 2017. ACCEPTED February 5, 2018.

INCLUDE WHEN CITING DOI: 10.3171/2018.2.FOCUS17543. 
evaluate the cost-effectiveness of TM care in comparison to routine in-person treatment of post-neurosurgical care patients using patient-perceived utility scores.

\section{Methods}

\section{Provider Center}

Established in 2001, the provider center, Sri Sathya Sai Institute of Higher Medical Sciences, located in Bengaluru, India, is a multispecialty charitable hospital that provides comprehensive care, including consultation, diagnosis, in-patient treatment, and follow-up to all patients, free of charge. The Department of Neurosurgery performs around 1800 elective (nontrauma) procedures every year that span neurooncology, cerebrovascular surgery, and skull base surgery, spine and pediatric neurosurgery. TM was established in 2007 as an extension of the outpatient facilities for neurosurgery and cardiology and has provided more than 10,000 consultations to patients across two nodal TM centers in the states of West Bengal and Odisha. A majority of the patients in both specialties hail from nonurban areas in these two states and have poor direct access to superspecialty care.

\section{Study Design}

Cost-effectiveness analysis (CEA) of the TM program was performed using data collected retrospectively from online records of 1200 consecutive post-neurosurgical care patients from West Bengal during a 52-month period from January 1, 2013, to April 30, 2017. All patients had undergone elective spinal or cranial surgery at the provider center. Following the first postoperative in-person visit within 2 weeks of their surgery, patients were advised to undergo follow-up at the TM nodal center at Barrackpore, West Bengal, within a period ranging from 3 months to 1 year after surgery. While 1034 of the study patients received at least one (including the first) follow-up consultation at the TM center as advised, the remaining 166 patients chose to visit the provider center for an in-person consultation for all consultations.

\section{Decision Model}

The decision-analytical model considered two options for providing outpatient consultation services for the remote population from West Bengal (Fig. 1). The first option of routine in-person care involved to-and-fro travel from West Bengal to the provider center in Bengaluru for a follow-up consultation. Because the distance involved is more than $1500 \mathrm{~km}$, the patient typically undergoes a 2-day train journey, arrives the day before the appointment, and stays at a hotel close to the provider center. The patient has either a single outpatient consultation or multiple consultations over a few days if she/he requires tests. She/he then travels back to West Bengal generally the day after the last consultation. The second option of TM care in the decision model involved receiving teleconsultations at the nodal center at Barrackpore, West Bengal, described below.

A TM consultation is considered successful if the patient goes home thereafter without requiring further evaluation or treatment; it is considered unsuccessful if he/

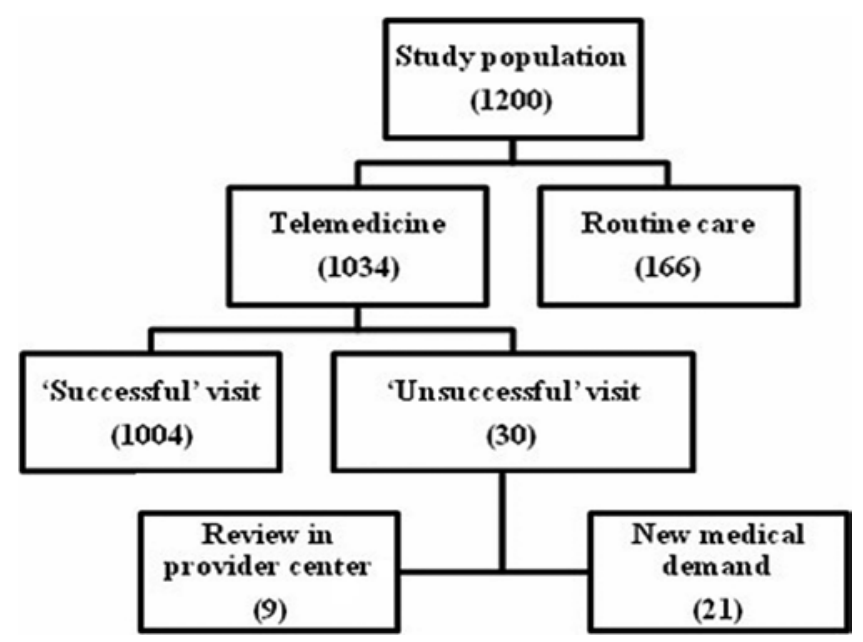

FIG. 1. Decision model used in our study. The number of patients in each component is listed within brackets.

she needs to undergo follow-up thereafter at the provider center for a complication or recurrence of her/his problem or at a local clinic for a nonneurosurgical problem (a "new medical demand").

\section{TM Center}

Over a 5- to 6-hour period weekly, around 20 patients receive real-time interactive consultations related to follow-up postoperative care at the TM nodal center. After having taken a prior appointment, the patient undergoes a consultation first with a trained local physician in his/her local language. The physician conducts a targeted neurological examination and registers investigation reports and new complaints. The neurosurgeon at the provider center then conducts a Skype teleconference with high-resolution image transmission that facilitates real-time exchange of data and images. The follow-up pertains to situations ranging from simple reassurance on clinical progress and return to work, to evaluating hormonal status for patients with suprasellar tumors, to giving advice on medications (e.g., tapering anticonvulsants or modifying them in case of side effects or nonresponse), to discontinuation of external bracing after a spinal instrumentation procedure. If the patient requires further evaluation or treatment at the provider center, she/he is then advised to come to Bengaluru for an in-person consultation. In case the patient requires emergent treatment, she or he is referred to a local hospital nearby.

\section{Study Assumption}

One of the key assumptions of our study was that there is no difference in the medical benefits for patients who received a TM consultation compared to those who received an in-person consultation at the provider center. This assumption is based on the findings of a previous article that reported good correlations between neurological findings picked up on-site and remotely ${ }^{22}$ and the fact that the same set of consultants catered to both groups in our study. With the consultation outcome thus remaining a constant across both groups in our study, the use of the widely accepted quality-adjusted life years as an outcome measure was not 
indicated. We instead used the patient's perceived utility score for their visit as an outcome measure.

\section{Utility Scores for Measuring Effectiveness}

We considered the patient's utility or preference for the type of health care access in 4 possible scenarios as the measure of effectiveness of the consultations. Scores on a scale of 0 to 10 ( 0 being worst and 10 being best) were obtained from a cohort of 100 consecutive patients from both the TM- and routine-care groups through a brief structured interview. The utility score for each scenario was an overall value based on the ease of accessing the center, quality of care, and overall experience. Hypothetical best-case and worst-case scenarios served as anchors to help guide the scoring. The mean scores for each of the 4 scenarios obtained from the patients yielded utility values that we then used for the analysis of the study cohort. A similar methodology for obtaining utility scores and effectiveness has been used previously. ${ }^{1}$

\section{Best-Case Scenario}

A patient receives an in-person neurosurgery consultation from a consultant from the provider center visiting their home town, obviating the need for the patient to travel to the provider center (hypothetical utility score: 1 ).

\section{Worst-Case Scenario}

No TM care is offered at the nodal center, with the patient essentially being refused access to services (hypothetical utility score: 0 ).

\section{Scenario \#1}

A patient receives a successful TM consultation as described above (utility score: 0.9 ).

\section{Scenario \#2}

The patient decides on receiving in-person neurosurgery consultation at the provider center in spite of the option of having a TM consultation (utility score: 0.8 ).

\section{Scenario \#3}

A patient receives an unsuccessful TM consultation in that he is asked to come down to the provider center for further management after the TM consultation (utility score: 0.6).

\section{Scenario \#4}

A patient comes to the TM center with a nonneurosurgical new medical demand and is asked to consult a local physician for the same. This is thus another unsuccessful TM consultation (utility score: 0.4 ).

\section{Cost Analysis Approach}

Analysis was performed using direct and indirect costs from a societal perspective-i.e., the analysis considered all costs irrespective of who the benefactors were. This is the recommended approach for CEAs in health care ${ }^{16}$ and is all the more relevant in the setting of a nonprofit system like that at the provider center.

\section{Costs Incurred by the TM Center}

The TM center operates in leased $500 \mathrm{sq} \mathrm{ft}$ premises with requisite furniture, computers and telecommunication facilities, and basic medical equipment. The teleconferencing system and connectivity were initially provided by the Indian Space Research Organization via a satellite communications system. ${ }^{8}$ From 2013 onward, this system was replaced by a personal computer-based high-definition audiovisual system using a 4-Mbps broadband connection provided by Bharat Sanchar Nigam Limited, a national telecommunications network. At the TM center, a general physician and the manager responsible for both technical and administrative operations are supported by two assistants. The cost of running this facility (including building rental, amortized cost of assets, payouts to personnel, cost of utilities including electricity and communication, and other miscellaneous costs) was calculated at market rates. The per annum depreciation rate for furniture was taken as $10 \%$, while that for office and medical equipment was taken as $20 \%$. Based on the percentage of neurosurgery to total TM consultations at the center during the 52-month study period, a prorated cost was calculated for arriving at the total TM facility cost.

\section{Costs Incurred by Patients}

Costs incurred by patients in obtaining TM consultation and in visiting the provider center were broadly categorized as those actually incurred and indirect costs arising from loss of potential earnings. All costing assumed that one attendant accompanied the patient for all visits.

Actual costs included the cost of consultation and investigations for the patient and cost of travel, board, and lodging. For each consultation (TM or routine), a cost of Indian rupee (INR) 135 per consultation was considered based on the latest Central Government Health Scheme (CGHS) rates (http://msotransparent.nic.in/writereaddata/ cghsdata/mainlinkfile/File816.pdf). As is the practice in corporate hospitals in India, the cost of only one consultation was assumed per episode even if there were multiple consultations. The costs of investigations were taken based on the CGHS rates for the same (Table 1). Second-class, 3-tier railway fares were considered for calculation of the cost of travel from the patients' home district to Barackpore or to Bengaluru. Local travel costs were taken based on actual local estimates of travel costs by bus. While the lodging cost at Barrackpore was nil (since none of the patients were required to stay overnight before or after the consultation), the same for Bengaluru was based on the average cost of stay at a one- or two-star hotel near the provider center. Food charges in either place were taken as the mean costs of three meals per day for two people.

Indirect cost or the cost of lost productivity was based on the following: 1) whether the patient is deemed to be employable (defined as no or mild impairments in vision, speech, hearing, and motor functions based on the latest national guidelines for the evaluation of various disabilities), ${ }^{18}$ 2) his/her profession, 3) the number of workdays lost (one per each TM visit; 4 travel days plus the days in Bengaluru from the first to the last consultation at the provider center), and 4) the latest minimum wages set by the Government for West Bengal (https://www.wblc.gov. 
TABLE 1. Cost per investigation in INR based on the CGHS rates (2014) and number of investigations compared in the two groups

\begin{tabular}{|c|c|c|c|}
\hline \multirow[b]{2}{*}{ Serial No. } & \multirow[b]{2}{*}{ Investigation } & \multicolumn{2}{|c|}{ Cost per Investigation (no. of times done ) } \\
\hline & & TM Care in West Bengal & Routine Care in Bengaluru \\
\hline 1 & Serum hormone level (T3, T4, TSH, \& cortisol) & $407(38)$ & 406 (1) \\
\hline 2 & Complete blood count & $122(0)$ & $122(1)$ \\
\hline 3 & Ultrasound of abdomen & $291(0)$ & $323(1)$ \\
\hline 4 & X-ray of cervical/thoracic/lumbar spine & $225(186)$ & $225(43)$ \\
\hline 5 & CT (plain) of cervical/thoracic/lumbar spine & $1350(0)$ & $1350(5)$ \\
\hline 6 & MRI (plain) of cervical/thoracic/lumbar spine & $2125(1)$ & $2125(10)$ \\
\hline 7 & MRI (contrast) of cervical/thoracic/lumbar spine & $4000(0)$ & $4000(3)$ \\
\hline 8 & CT (plain) of brain & $810(26)$ & $893(3)$ \\
\hline 9 & $\mathrm{CT}$ (contrast) of brain & $1215(12)$ & $1215(5)$ \\
\hline 10 & MRI (plain) of brain & 1998 (1) & $1998(0)$ \\
\hline 11 & MRI (contrast) of brain & $2848(6)$ & $2848(4)$ \\
\hline 12 & Nerve conduction study & $638(0)$ & $638(1)$ \\
\hline Total cost of investigations & & $114,167(270)$ & $71,296(77)$ \\
\hline
\end{tabular}

$\mathrm{TSH}=$ thyroid-stimulating hormone.

in/sites/default/files/synopsys/01-07-2016/synopsis-ofminimum-rates-of-wages-in-scheduled-employments $\% 20$ in-west-bengal-as-on-01-07-2016.pdf). For housewives, a cost of INR 100 per day was assumed to have been incurred as reimbursement to someone for doing their work. For students, this cost was taken as nil.

\section{Statistical Analysis}

Cost-effectiveness was measured for the 2 options: 1) patients choosing TM consultations and 2) patients choosing routine care. Cost-effectiveness was defined as the total cost incurred by each group divided by the effectiveness for that group, where effectiveness $=$ number of patients $\times$ utility.

The mean patient-perceived utility value for each of the 4 scenarios was used to calculate the effectiveness of the consultations by using the above formula. As an example, if 100 patients received successful TM consultations and the utility of a successful TM consultation is 0.9 , then the TM effectiveness is the following: $100 \times 0.9=90$. The incremental cost-effectiveness ratio (ICER) represents the mean incremental cost associated with one additional unit of the measure of effectiveness. This was calculated as the

TABLE 2. Cost breakdown in INR for the two model options

\begin{tabular}{lrrrrr}
\hline & \multicolumn{2}{c}{ TM Care } & & \multicolumn{2}{c}{ Routine Care } \\
\cline { 2 - 3 } \cline { 5 - 6 } Cost Component & $\begin{array}{c}\text { Cost } \\
(n=1034)\end{array}$ & $\begin{array}{c}\text { Mean Cost } \\
\text { per Episode }\end{array}$ & & $\begin{array}{c}\text { Cost } \\
(n=166)\end{array}$ & $\begin{array}{c}\text { Mean Cost } \\
\text { per Episode }\end{array}$ \\
\hline $\begin{array}{l}\text { Prorated capital \& } \\
\text { personnel }\end{array}$ & $1,275,236$ & 1233 & & 22,410 & 135 \\
\hline Travel \& lodging & 994,567 & 961 & & 651,459 & 3924 \\
\hline Lost productivity & 33,995 & 34 & & 164,344 & 990 \\
\hline Investigations & 114,167 & 110 & & 71,296 & 430 \\
\hline Total cost incurred & $2,417,965$ & 2338 & & 909,509 & 5479 \\
\hline
\end{tabular}

difference in cost per patient in the two groups $(\mathrm{C} 1-\mathrm{C} 2)$ divided by the difference in the effectiveness per episode $(\mathrm{E} 1$ - E2)-i.e., $\mathrm{ICER}=(\mathrm{C1}-\mathrm{C} 2) /(\mathrm{E} 1$ - E2). The incremental cost and effectiveness values were plotted in a 4-quadrant graph to determine the cost-effectiveness plane as has been described previously. ${ }^{13}$

One-way sensitivity analysis was performed by varying the base-case values of all the model parameters across a wide range while keeping all the other parameters constant. A realistic range for variation in each parameter was identified by using either maximum or minimum values, one standard deviation above and below the mean value, or the best-case and worst-case scenarios. For calculation of cost per kilometer travelled by the patients for a consultation, a weighted mean of the total cost incurred by both the TM- and routine-care groups was considered. A tornado analysis resulting from the one-way sensitivity analyses provided information about the relative influence of key variables on the cost-effectiveness ratios of the TM- and routine-care arms in the model and identified thresholds where the optimal care strategy changes.

\section{Results}

\section{Clinical Profile}

While patients' spinal pathologies constituted only $56 \%$ of patients in the TM group, they accounted for $90.9 \%$ (p $<0.0001$ ) of patients in the routine-care group. The corresponding figures for cranial pathologies were $44 \%$ and $9 \%$, respectively $(\mathrm{p}<0.0001)$. Nine patients were examined in the provider center following unsuccessful TM visits: 5 had cranial pathologies and 4 spinal. Twenty-one patients visited the TM center with new medical demands pertaining to nonneurosurgical specialties.

\section{Costs Incurred}

Table 2 lists the total costs incurred by patients and providers in the 4 different scenarios. The 1) sum of the 
TABLE 3. Base-case results of the cost-effectiveness analysis

\begin{tabular}{|c|c|c|c|c|}
\hline \multirow[b]{2}{*}{ Variable } & \multicolumn{3}{|c|}{ TM Care } & \multirow{2}{*}{$\begin{array}{l}\text { Routine Care } \\
\text { Scenario \#2 }\end{array}$} \\
\hline & Scenario \#1 & Scenario \#3 & Scenario \#4 & \\
\hline No. of patients & 1004 & 9 & 21 & 166 \\
\hline Utility score & 0.9 & 0.6 & 0.4 & 0.8 \\
\hline Effectiveness (no. of patients $\times$ utility score) & 903.6 & 5.4 & 8.4 & 132.8 \\
\hline Efficiency (\% of successful visits) & & $97 \%$ & & $100 \%$ \\
\hline Overall effectiveness & & 917.4 & & 132.8 \\
\hline Cost per patient episode (INR) & & 2338 & & 5479 \\
\hline Overall utility score & & 0.89 & & 0.80 \\
\hline Cost-effectiveness ratio (INR per unit of perceived utility) & & 2635 & & 6848 \\
\hline ICER & \multicolumn{4}{|c|}{$(2338-5479 / 0.89-0.80)=-34,900 ;$ dominant $^{*}$} \\
\hline
\end{tabular}

* ICER value is in the southeast quadrant of the cost-effectiveness graph in Fig. 2 (i.e., TM care dominates routine care).

prorated costs relating to capital and personnel and 2) total costs pertaining to travel, investigations, and lost productivity in the TM-care and routine-care groups over 52 months were INR 2,417,965 and INR 909,509, respectively. The mean per episode cost was INR 2338 for TM care versus INR 5479 for routine care (Table 2).

\section{Base-Case Results of the CEA}

The TM-care success rate was 97\%, with 1004 patients having met their medical need completely after the TM consultation. The overall TM utility was $89 \%$, whereas that of the provider center consultations was lower at $80 \%$. The overall effectiveness of the TM-care group was 917.4
$(1004 \times 0.90+9 \times 0.60+21 \times 0.40)$ and that of routine care was $132.8(166 \times 0.8)$ (Table 3$)$. The cost-effectiveness ratios were INR $2635(2,417,965 / 917.4)$ and INR $6848(909,509 / 132.8)$ for the TM-care and routine-care groups, respectively. The ICER value was calculated to be $-34,900$ INR/unit of effectiveness (2338 - 5479/0.89 -0.80 ). This was located in the southeast quadrant of the cost-effectiveness plane (Fig. 2), indicating that TM care dominated routine care in our analysis (Table 3 ).

\section{One-Way Sensitivity Analysis}

On the basis of this analysis (Table 4) using low and high values, the 4 most influential variables that could po-

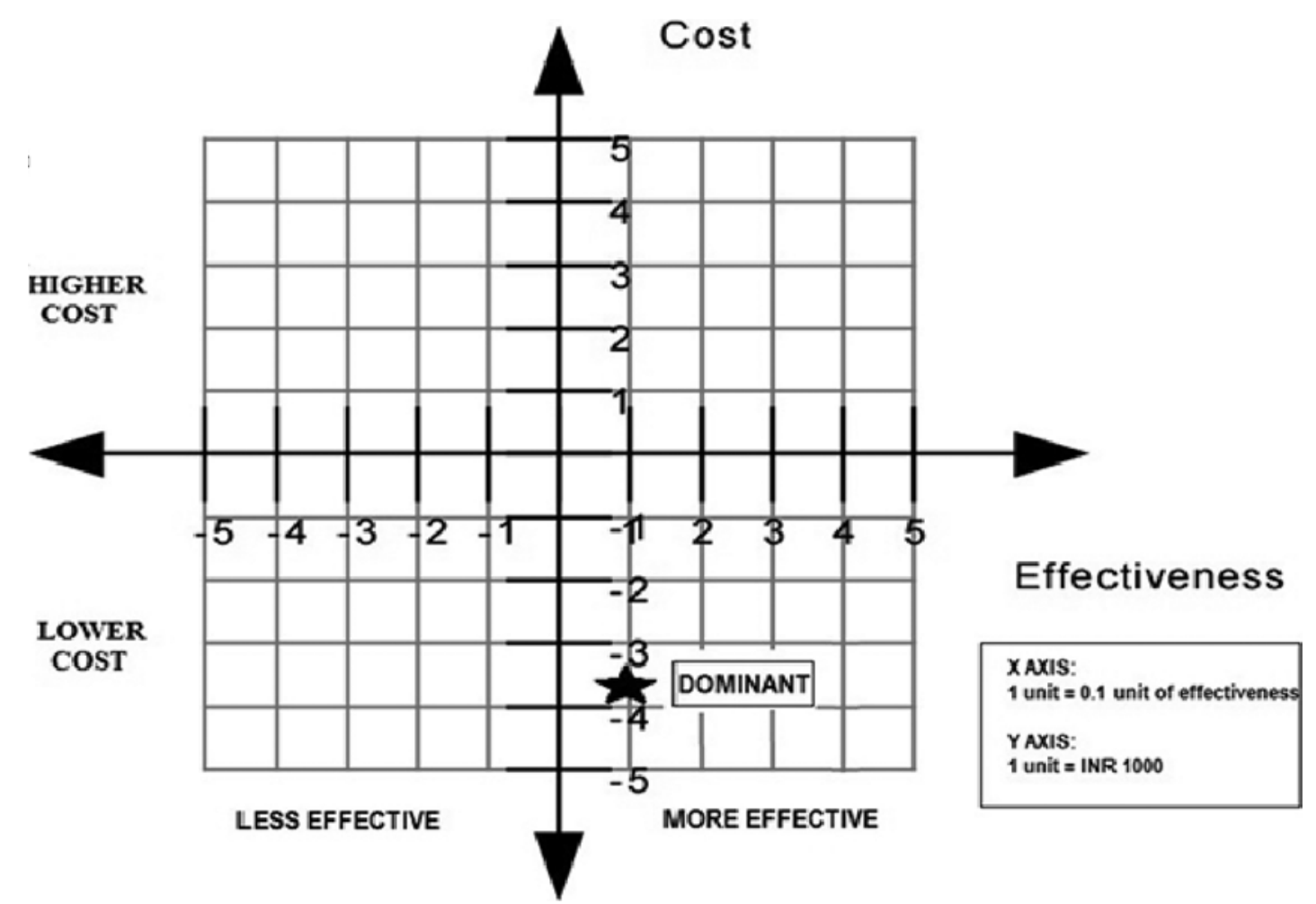

FIG. 2. Four-quadrant graph to demonstrate the plane of cost-effectiveness. In this study, the TM-care strategy (marked with a star) dominated routine care, with the incremental cost-effectiveness value being located in the southeast quadrant of the graph . 
TABLE 4. One-way sensitivity and threshold analyses

\begin{tabular}{lcccc}
\hline \multicolumn{1}{c}{ Variable } & Base Case & Low Value & High Value & Threshold \\
\hline Efficiency (success rate) of TM & $97 \%$ & $0 \%$ & $100 \%$ & $44 \%$ \\
\hline Utility of TM consultation & 0.89 & 0.001 & 2068 & 406 \\
\hline No. of patients visiting TM & 1034 & 200 & $100 \%$ & NA \\
\hline Prorated \% of neurosurgery TM & $48 \%$ & $5 \%$ & 9 & NA \\
\hline Workdays lost in routine care & 7 & 5 & $1,725,780$ & NA \\
\hline TM facility cost/annum (INR) & 547,780 & 399,780 & 3000 & 820 \\
\hline Distance travelled $(\mathrm{km})$ & 177 & 17 & & \\
\hline
\end{tabular}

$\mathrm{NA}=$ not applicable.

tentially affect cost-effectiveness were as follows: 1) number of patient visits in TM, 2) utility of the TM consultation, 3) travel distance of the patients to the TM center, and 4) success rate of TM. Threshold values for these variables were calculated to identify when TM would cease to be more cost-effective than routine care (Table 4). The tornado analysis (Fig. 3) provides information about the relative influence of the 4 key variables on the cost-effectiveness ratio in our model.

\section{Discussion}

\section{Cost-Related Studies in TM}

The ready availability of newer communication platforms,${ }^{15}$ high convenience factor, and travel and time cost savings associated with TM have resulted in its increasing usage in health care. There is, however, a dearth of robust cost-effectiveness studies in TM literature, with many of the cost-related studies being reportedly of poor quality. ${ }^{7,19,23}$ A recent systematic review found the efficacy of TM to vary from inconsistent to potentially beneficial. ${ }^{10}$ Some recent studies have demonstrated TM to be costineffective ${ }^{5,9,11}$ challenging the intuitive notion that TM is universally cost-effective. The unsettling results of these studies were attributed to cost-intensive TM interventions ${ }^{11}$ or to new usage of medical services via a direct-to-consumer model that facilitates easy access to health care. ${ }^{5}$

\section{Outcome Measures Used in TM}

Various methods of evaluating TM studies have included economic evaluations, patient satisfaction levels, and clinical outcomes. ${ }^{2}$ Patient-perceived scores for the type and utility of the health care access that they received have been used previously, albeit from a physician's perspective. ${ }^{1}$ Patient-perceived preference-based measures, similar to the one that we have used in this study, have been used previously in the Indian population ${ }^{20,26}$ in other post-neurosurgical care contexts.

\section{Importance of Follow-Up Care in Neurosurgery}

The maintenance of a successful follow-up after neurosurgical intervention is essential to justify the investment that super specialty care entails. In the developing world and in underserved areas in the more developed nations, transportation, limited medical resources, and, occasionally, social stigma can pose significant challenges with re- gard to diagnosis and follow-up care of patients with neurosurgical disease. ${ }^{6} \mathrm{TM}$ can help in overcoming some of these limitations by cutting down on the travel time and travel expenses incurred by the patient during follow-up care, but they remain largely unexplored in elective neurosurgery.

\section{Telemedicine in Neurosurgery}

Most of the studies in neurosurgery-related TM care have addressed the utility of TM in time-sensitive scenarios like stroke, ${ }^{24,29}$ epilepsy, ${ }^{14,27}$ and trauma. ${ }^{3,4,17}$ The feasibility of TM care has also been recently demonstrated for pediatric neurosurgical problems $\mathrm{s}^{12}$ and in the follow-up care of elective neurosurgical patients. ${ }^{8,21}$ While the benefits of TM for postoperative care have been evaluated in various general surgical conditions of low complexity, ${ }^{28}$ the sustainability of such a TM program from an economic standpoint has not been analyzed in a neurosurgical postoperative cohort. While we had previously reported our findings of a simple cost analysis of a large neurosurgical patient cohor ${ }^{8}$ served by the same provider center, the current CEA study is unique in its use of patient-perceived utility scores as a measure of effectiveness.

\section{Interpretation of the One-Way Sensitivity Results}

Interestingly, even if around half the TM consultations proved to be unsuccessful in our cohort, TM care would still remain more cost-effective than routine care. However, if the total number of patients utilizing our TM facility was to decrease by more than half, or if the patients' travel distance to the TM center was to increase by 5 times, or if patients' mean utility score for TM decreased to less than 0.3 for reasons that could affect the overall experience of their consultation (e.g., a low-quality teleconferencing system, understaffing or inefficient management of the TM services, or a high percentage of new medical demands), then TM would cease to be more cost-effective than routine care.

\section{New Medical Demand}

A recent large study in the US has demonstrated directto-consumer TM care to be cost-ineffective primarily because of new medical demands in as many as $80 \%$ of the patients. ${ }^{5}$ In our study, the percentage of patients who presented in the TM center with nonneurosurgical problems was only around $2 \%$. If this percentage was to increase sig- 


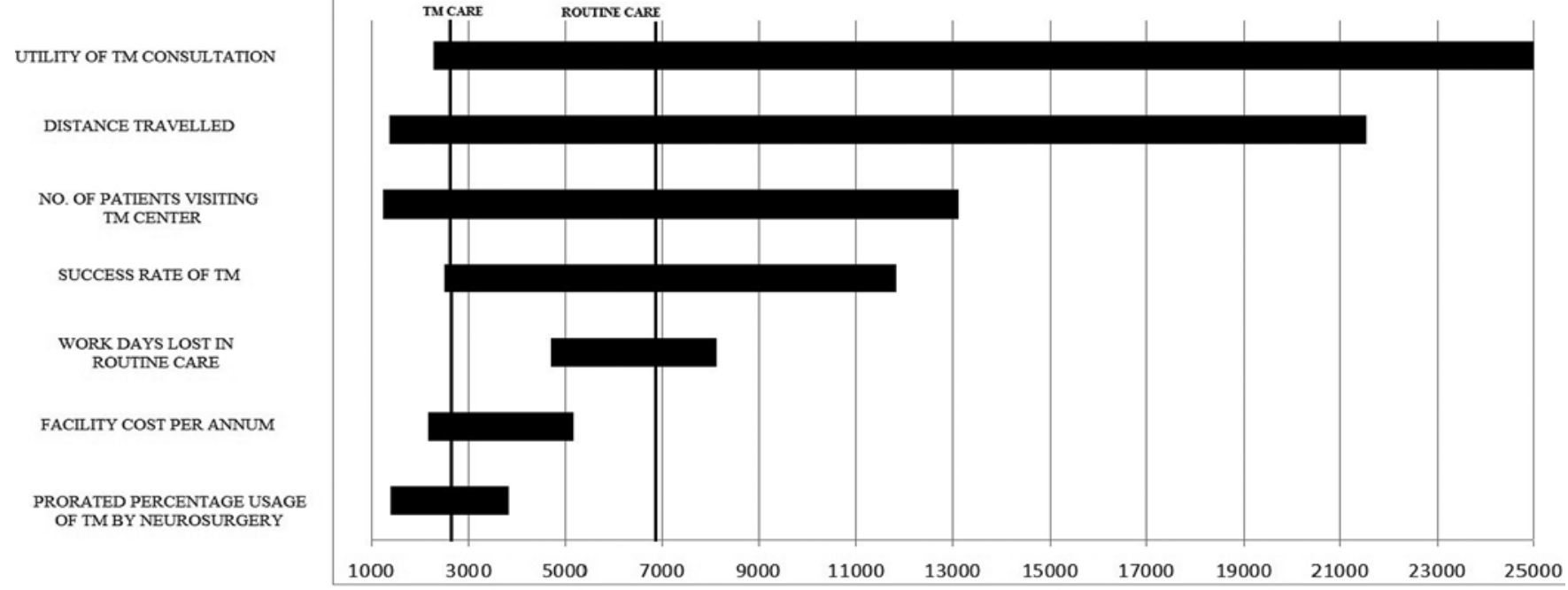

COST EFFECTIVENESS RATIO

FIG. 3. Tornado diagram depicting the relative effect of key variables on the cost-effectiveness ratio of TM care determined by sensitivity analysis.

nificantly, the overall utility and, hence, cost-effectiveness of TM would decrease. It is thus imperative that patients understand the scope of the TM services well at the time of discharge from the hospital.

\section{Cost-Effectiveness Plane of TM Care}

There has been growing interest in the concept of the cost-effectiveness plane in CEA. As most new treatments are thought to deliver more value or effectiveness at increased costs ${ }_{13}^{13}$ most of the studies in the western population pertain to cost-effectiveness in the northeast quadrant in the 4-quadrant graph alluded to earlier. The maximum acceptable ICER based on willingness to pay was developed for this effective but cost-intensive quadrant. Incremental cost-effectiveness values in the southeast quadrant, like those of TM care in our cohort, are reflective of more effectiveness delivered at a lower cost.

\section{Implications of Our Study}

TM for follow-up care for elective neurosurgical care patients is a potentially viable, cost-effective option if the provider center sets up a TM center close to a well-served remote region, and if patients are well educated about the scope and utility of the TM services. It is expected that the presence of comorbidities, infections, or wound-related problems as well as early recurrences in intracranial pathologies that carry a poor prognosis would predispose to higher rates of unsuccessful follow-up TM consultations.

\section{Study Limitations}

Though selection bias in our study was avoided by recruiting consecutive follow-up patients in the study period, the study is weighed down by the inherent limitations of a retrospective study. The heterogeneous clinical profile of the patients in the 2 groups could have been a source of bias. The utility scores were based on a sample population, and this could influence the results if there was greater variance between scenarios. It would have been useful to explore the change in these scores over an extended followup period. The use of the first follow-up episodes rather than a longitudinal approach to the analysis could potentially affect results. There could also be potential sources of over- and underestimation of costs across both groups for factors such as travel and lodging.

\section{Conclusions}

TM care dominates the in-person care strategy by providing more effective and less expensive follow-up care for a remote post-neurosurgical care population in India. In our setting, this benefit of TM is sustainable even if half the TM consultations turn out to be unsuccessful. The viability of TM as a cost-effective care protocol is attributed to a combination of factors, such as an adequate patient volume utilizing TM, patient utility, success rate of TM, and the patients' travel distance to the TM center.

\section{References}

1. Agha Z, Schapira RM, Maker AH: Cost effectiveness of telemedicine for the delivery of outpatient pulmonary care to a rural population. Telemed J E Health 8:281-291, 2002

2. AlDossary S, Martin-Khan MG, Bradford NK, Smith AC: A systematic review of the methodologies used to evaluate telemedicine service initiatives in hospital facilities. Int J Med Inform 97:171-194, 2017

3. Ashkenazi I, Haspel J, Alfici R, Kessel B, Khashan T, Oren M: Effect of teleradiology upon pattern of transfer of head injured patients from a rural general hospital to a neurosurgical referral centre. Emerg Med J 24:550-552, 2007

4. Ashkenazi I, Zeina AR, Kessel B, Peleg K, Givon A, Khashan T, et al: Effect of teleradiology upon pattern of transfer of head injured patients from a rural general hospital to a neurosurgical referral centre: follow-up study. Emerg Med J 32:946-950, 2015

5. Ashwood JS, Mehrotra A, Cowling D, Uscher-Pines L: Direct-to-consumer telehealth may increase access to care 
but does not decrease spending. Health Aff (Millwood) 36:485-491, 2017

6. Bartolozzi A: Post-operative responsibilities of charitable neurosurgery clinics in global settings. Harvard Medical Student Review. March 2, 2014. (http://hmsreview.org/ issue/2014/4/post-operative-responsibilities-of-charitableneurosurgery-clinics-in-global-settings) [Accessed March 5, 2018]

7. Bergmo TS: Can economic evaluation in telemedicine be trusted? A systematic review of the literature. Cost Eff Resour Alloc 7:18, 2009

8. Dadlani R, Mani S, Jai Ganesh AU, Mohan D, Rajgopalan N, Thakar S, et al: The impact of telemedicine in the postoperative care of the neurosurgery patient in an outpatient clinic: a unique perspective of this valuable resource in the developing world-an experience of more than 3000 teleconsultations. World Neurosurg 82:270-283, 2014

9. Dixon P, Hollinghurst S, Edwards L, Thomas C, Foster A, Davies B, et al: Cost-effectiveness of telehealth for patients with depression: evidence from the Healthlines randomised controlled trial. BJPsych Open 2:262-269, 2016

10. Ekeland AG, Bowes A, Flottorp S: Effectiveness of telemedicine: a systematic review of reviews. Int J Med Inform 79:736-771, 2010

11. Henderson C, Knapp M, Fernández JL, Beecham J, Hirani SP, Cartwright M, et al: Cost effectiveness of telehealth for patients with long term conditions (whole systems demonstrator telehealth questionnaire study): nested economic evaluation in a pragmatic, cluster randomised controlled trial. BMJ 346:f1035, 2013

12. James HE: Pediatric neurosurgery telemedicine clinics: a model to provide care to geographically underserved areas of the United States and its territories. J Neurosurg Pediatr 25:753-757, 2016

13. Klok RM, Postma MJ: Four quadrants of the cost-effectiveness plane: some considerations on the south-west quadrant. Expert Rev Pharmacoecon Outcomes Res 4:599-601, 2004

14. Larner AJ: Teleneurology: an overview of current status. Pract Neurol 11:283-288, 2011

15. Ma Y, Jones G, Tay YK, Hunter T, Holden D, Rodgers-Wilson S, et al: Post-operative telephone review is safe and effective: prospective study - Monash outpatient review by phone trial. ANZ J Surg [epub ahead of print], 2017

16. Mair FS, Haycox A, May C, Williams T: A review of telemedicine cost-effectiveness studies. J Telemed Telecare 6 (Suppl 1):S38-S40, 2000

17. Migliaretti G, Ciaramitaro P, Berchialla P, Scarinzi C, Andrini R, Orlando A, et al: Teleconsulting for minor head injury: the Piedmont experience. J Telemed Telecare 19:33-35, 2013

18. Ministry of Social Justice and Empowerment (India): Guidelines for Evaluation of Various Disabilities and Procedure for Certification. New Dehli: Gazette of India, 2001 (http://disabilityaffairs.gov.in/upload/uploadfiles/files/ guidelines\%202001_compressed.pdf) [Accessed March 5, 2018]

19. Polisena J, Coyle D, Coyle K, McGill S: Home telehealth for chronic disease management: a systematic review and an analysis of economic evaluations. Int J Technol Assess Health Care 25:339-349, 2009

20. Rajshekhar V, Muliyil J: Patient perceived outcome after central corpectomy for cervical spondylotic myelopathy. Surg Neurol 68:185-191, 2007
21. Reider-Demer M, Raja P, Martin N, Schwinger M, Babayan D: Prospective and retrospective study of videoconference telemedicine follow-up after elective neurosurgery: results of a pilot program. Neurosurg Rev [epub ahead of print], 2017

22. Riedel CJ, Choudhri TF, Wilson D, Khanafer N, Alaoui A, Tohme W, et al: Telemedicine in neurosurgery: peri-operative management, in Nelson R, Gelish A, Mun SK (eds): Proceedings. Pacific Medical Technology Symposium (PACMEDTek). Los Alamitos, CA: IEEE Computer Society, 1998, pp 80-82

23. Rojas SV, Gagnon MP: A systematic review of the key indicators for assessing telehomecare cost-effectiveness. Telemed J E Health 14:896-904, 2008

24. Sharma R, Fleischut P, Barchi D: Telemedicine and its transformation of emergency care: a case study of one of the largest US integrated healthcare delivery systems. Int J Emerg Med 10:21, 2017

25. Sinha VD, Tiwari RN, Kataria R: Telemedicine in neurosurgical emergency: Indian perspective. Asian J Neurosurg 7:75-77, 2012

26. Thakar S, Rajshekhar V: Evaluation of pain as a preferencebased health status measure in patients with cervical spondylotic myelopathy undergoing central corpectomy. Acta Neurochir (Wien) 154:335-340, 2012

27. Velasquez SE, Chaves-Carballo E, Nelson EL: Pediatric teleneurology: a model of epilepsy care for rural populations. Pediatr Neurol 64:32-37, 2016

28. Vella MA, Kummerow Broman K, Tarpley JL, Dittus RS, Roumie CL: Postoperative telehealth visits: assessment of quality and preferences of veterans. JAMA Surg 150:11971199, 2015

29. Wechsler LR, Demaerschalk BM, Schwamm LH, Adeoye $\mathrm{OM}$, Audebert HJ, Fanale CV, et al: Telemedicine quality and outcomes in stroke: a scientific statement for healthcare professionals from the American Heart Association/American Stroke Association. Stroke 48:e3-e25, 2017

\section{Disclosures}

The authors report no conflict of interest concerning the materials or methods used in this study or the findings specified in this paper.

\section{Author Contributions}

Conception and design: Thakar, Mani, Shyam. Acquisition of data: Rajagopal, Rao, Srinivasa. Analysis and interpretation of data: Thakar. Drafting the article: Thakar, Shyam. Critically revising the article: Thakar, Aryan, Rao. Reviewed submitted version of manuscript: all authors. Approved the final version of the manuscript on behalf of all authors: Thakar. Statistical analysis: Mani. Administrative/technical/material support: Mohan. Study supervision: Hegde.

\section{Supplemental Information \\ Videos}

Video Abstract. https://vimeo.com/262374146.

\section{Correspondence}

Sumit Thakar: Sri Sathya Sai Institute of Higher Medical Sciences, Bangalore, India.sumit.thakar@gmail.com. 